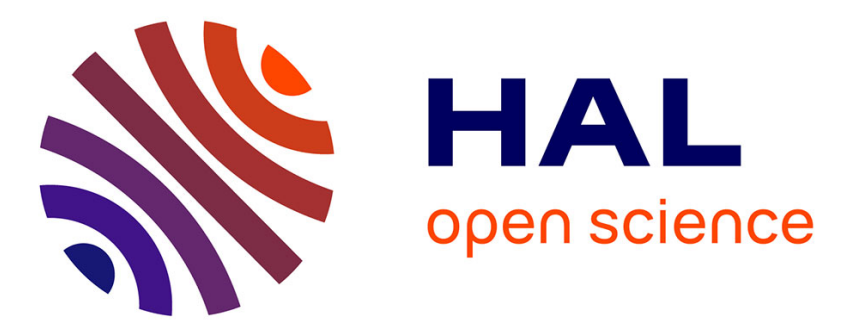

\title{
Localisation et caractérisation semi-automatique des géomorphosites fluviaux potentiels. Exemples d'applications à partir d'outils géomatiques dans le bassin de la Drôme (France)
}

\author{
Elise Wiederkehr, Simon Dufour, Hervé Piégay
}

\section{To cite this version:}

Elise Wiederkehr, Simon Dufour, Hervé Piégay. Localisation et caractérisation semi-automatique des géomorphosites fluviaux potentiels. Exemples d'applications à partir d'outils géomatiques dans le bassin de la Drôme (France). Géomorphologie: relief, processus, environnement, 2010, 2, pp.175-188. 10.4000/geomorphologie.7912 . hal-00828810

\section{HAL Id: hal-00828810 https://hal.science/hal-00828810}

Submitted on 23 Oct 2013

HAL is a multi-disciplinary open access archive for the deposit and dissemination of scientific research documents, whether they are published or not. The documents may come from teaching and research institutions in France or abroad, or from public or private research centers.
L'archive ouverte pluridisciplinaire HAL, est destinée au dépôt et à la diffusion de documents scientifiques de niveau recherche, publiés ou non, émanant des établissements d'enseignement et de recherche français ou étrangers, des laboratoires publics ou privés. 

processus, environnement

2/2010 (2010)

Varia

Elise Wiederkehr, Simon Dufour et Hervé Piégay

\title{
Localisation et caractérisation semi- automatique des géomorphosites fluviaux potentiels. Exemples d'applications à partir d'outils géomatiques dans le bassin de la Drôme (France)
}

\begin{abstract}
Avertissement
Le contenu de ce site relève de la législation française sur la propriété intellectuelle et est la propriété exclusive de l'éditeur.

Les œuvres figurant sur ce site peuvent être consultées et reproduites sur un support papier ou numérique sous réserve qu'elles soient strictement réservées à un usage soit personnel, soit scientifique ou pédagogique excluant toute exploitation commerciale. La reproduction devra obligatoirement mentionner l'éditeur, le nom de la revue, l'auteur et la référence du document.

Toute autre reproduction est interdite sauf accord préalable de l'éditeur, en dehors des cas prévus par la législation en vigueur en France.
\end{abstract}

\section{revues.org}

Revues.org est un portail de revues en sciences humaines et sociales développé par le Cléo, Centre pour l'édition électronique ouverte (CNRS, EHESS, UP, UAPV).

Référence électronique

Elise Wiederkehr, Simon Dufour et Hervé Piégay, « Localisation et caractérisation semi-automatique des géomorphosites fluviaux potentiels. Exemples d'applications à partir d'outils géomatiques dans le bassin de la Drôme (France) 》, Géomorphologie : relief, processus, environnement [En ligne], 2/2010 | 2010, mis en ligne le 01 juillet 2012, consulté le 12 octobre 2012. URL : http://geomorphologie.revues.org/7912 ; DOI : 10.4000/ geomorphologie. 7912

Éditeur : Groupe français de géomorphologie

http://geomorphologie.revues.org

http://www.revues.org

Document accessible en ligne sur : http://geomorphologie.revues.org/7912

Ce document est le fac-similé de l'édition papier.

(c) Groupe français de géomorphologie 


\title{
Localisation et caractérisation semi-automatique des géomorphosites fluviaux potentiels. Exemples d'applications à partir d'outils géomatiques dans le bassin de la Drôme (France) \\ Location and semi-automatic characterisation of potential fluvial geomorphosites. Examples of applications from geomatic tools in the Drôme River basin (France)
}

\author{
Elise Wiederkehr*, Simon Dufour**, Hervé Piégay*
}

\begin{abstract}
Résumé
Les réseaux hydrographiques abritent des géomorphosites de qualité, comme le sont les gorges et les secteurs à lit mobile ou en tresses. Des outils de caractérisation physique des corridors fluviaux ont été mis en place pour les localiser de façon systématique. Nous nous sommes orientés vers des outils géomatiques afin d'éviter un travail de terrain long et fastidieux et obtenir un résultat homogène à l'échelle du bassin hydrographique. Deux exemples de géomorphosites potentiels sont détaillés, chacun s'appuyant sur une approche spécifique. Dans un premier temps, l'utilisation de couches vectorielles et d'un MNT ont permis de décrire la géométrie des plaines alluviales et de détecter les secteurs en gorges. Dans un second temps, nous avons couplé les données extraites d'orthophotographies avec des informations vectorielles afin de caractériser les styles fluviaux et, in fine, identifier les zones à haute valeur biogéomorphologique comme les secteurs en tresses. Une série de tests a été effectuée dans le bassin de la Drôme pour expérimenter le processus d'extraction de l'information à partir de photographies à très haute résolution et de modèles numériques de terrain et pour évaluer le potentiel de ces données en termes d'indicateurs de caractérisation physique des réseaux hydrographiques (structure paysagère, morphologie, conditions hydrauliques). Ainsi, les procédures mises en place ont permis d'identifier et de caractériser et ce, de façon systématique et rapide à l'échelle du continuum fluvial, les différentes formes de fonds de vallée et les différents types de tracés en plan du lit, afin de localiser les secteurs dont les propriétés esthétiques et patrimoniales permettent de les considérer comme des « géomorphosites ».
\end{abstract}

Mots clés : géomorphosites, outils géomatiques, style fluvial, écotone, indicateurs.

\begin{abstract}
The hydrographic networks can provide geomorphosites of high quality such as gorges, river corridors with shifting channels or braided pattern. To identify them, we developed tools for characterising biophysical features of the valley bottoms. We developed geomatic tools in order to avoid fieldwork, which is a long and tedious work and to achieve homogeneous data throughout a network covering an entire region or a country. Two approaches have been tested. On the one hand, GIS layers and DEM were used to describe the geometry of the alluvial plains and to target gorges reaches. On the other hand, we coupled orthophotos provided by IGN (National Geographical Institute) to vectorial layers to understand the fluvial patterns and to identify reaches of high environmental values, such as braided reaches. We did a series of tests on the River Drôme (France) to assess the feasibility of extracting information from high-resolution photographs so as to set up indicators for the physical characterisation of hydrographic networks. The data thus obtained was used to identify, characterise and locate the valley and fluvial corridor patterns, including reaches of quality corresponding to the so-called 'geomorphosites'.
\end{abstract}

Key words: geomorphosites, geomatic tools, fluvial pattern, ecotone, indicators.

\section{English abridged version}

'Geomorphosites' are geomorphological features with 'strong interest' for ecological, aesthetic, cultural, economic and also educational reasons (Panizza 2001; Panizza and Piacente, 1993, 2003; Reynard 2005a). Hydrographic networks present some interesting geomorphosites, such as gorges, waterfalls, braided areas, marshes, and oxbow lakes. To preserve and re-

\footnotetext{
* CNRS UMR 5600 Environnement, Ville et Société - Université de Lyon, ENS de Lyon - 15, parvis René Descartes - BP 7000 - 69342 LYON Cedex 07 - France. Courriel : elise.wiederkehr@ens-lyon.fr

** COSTEL UMR CNRS 6554 LETG - Université Rennes 2 - Département de Géographie - Place du Recteur Henri Le Moal - 35043 RENNES Cedex

- France. Courriel : simon.dufour@uhb.fr
} 
habilitate these landscape features, it is necessary to locate them at regional scale. This can be achieved thanks to maps, tourist guides or field survey. But the achievement of an exhaustive inventory across a hydrographic network as large as that of the Rhône (e.g., 45,000 km long) requires the development of tools to extract indicators automatically or semi-automatically. The aim of this article is to highlight some geomatic methods based on recent remote sensing techniques to map geomorphosites. Two complementary examples are described to illustrate the approach at network scale. On one hand, we used GIS layers and a DEM to describe the geometry of the alluvial plains to target areas with gorges. On the other hand, we coupled orthophotos to vectorial layers to characterise the fluvial patterns and so identify areas of high ecological value such as braided reaches. The Drôme main stem was chosen as the test reach because it is characterised by contrasting landscapes with braided, dyked, gorges or straight reaches alternating over a $100 \mathrm{~km}$ long reach (Piégay and Landon, 1997).

Gorge reaches correspond to narrow and deep valleys with relatively steep slopes. To detect gorge reaches, three steps were considered: i) detection of homogeneous geomorphological reaches based on a threshold statistical test (Clément and Piégay, 2003); ii) calculation of a valley confinement index which is the ratio between the width of the active channel and the width of the valley bottom for each of the identified geomorphic reaches; iii) characterisation of valley geometry on selected narrow valley reaches (i.e., the ones with a confinement index lower than 0.3) based on metrics extracted from a set of buffers (e.g., valley depth, valley width at the top). The determination of fluvial pattern was based on four metrics: $i$ ) the average width of the active channel normalised by the size of the watershed area; ii) the confinement index, which gives an indication of the potential mobility of the river; iii) the average surface water channel/active channel ratio which provides information on the diversity of environments of the active channel (main channel, secondary channels, etc.; (Alber and Piégay, in press); iv) the index of sinuosity of the river which is the ratio of the length of the active channel centre line divided by the length of the valley bottom centre line. An ascending hierarchical classification (fig. 3) was performed on a data set of 53 reaches described by four variables (Lebart et al., 1995). We then studied the statistical distribution (median, quartiles) of each metric class in order to select five fluvial pattern types (single-bed channel to braided reaches. This allowed us to locate the geomorphosites (fig. 4). We developed another method to identify geomorphosites with high ecological value using an index of ecotone density (i.e., transition zone between two ecosystems that play an important role in maintaining biodiversity). We have identified three types of ecotones: aquatic/bar, bar/arboreous vegetation, and finally arboreous vegetation/aquatic environment. Then, we mapped (fig. 5) the density of each ecotone by homogeneous geomorphic reach.

The methodological framework that we have developed to identify potential geomorphosites could also be used to implement the Water Framework Directive (WFD; Wasson, 2001;
Roche et al., 2004; Porcher, 2009). Each water body can be characterised semi-automatically by a set of metrics defined by the operator. The database contains continuous information over an entire network. However the extraction of indicators from images has constraints because of image resolution $(50 \mathrm{~cm})$ and spatial variations in image radiometry, limiting efficiency of some extraction procedures. For example, we can study the lateral mobility or fish habitat to improve the existing models (Ginot, 1998; Waddle, 2001; Lamouroux, 2002), or to develop models of regionalised distribution of species and habitats.

\section{Introduction}

\section{Géomorphosites et réseaux hydrographiques}

Le paysage géomorphologique, tel que l'entend E. Reynard (2005a), correspond à une portion du relief terrestre à la fois vue, perçue et exploitée par l'Homme, reflétant le fruit d'un incessant travail de processus qui ont forgé sa forme actuelle et qui seront également à l'origine de son évolution future. Au sein d'un paysage géomorphologique, certains auteurs identifient des « géomorphosites » (Panizza, 2001), ceux-ci étant définis comme des sites dont les propriétés géomorphologiques présentent un intérêt social. Le non-initié n'emploiera pas le mot de « géomorphosite » mais préférera qualifier tel ou tel site d'exceptionnel, de spectaculaire, voire de grandiose. Il ne suffit cependant pas d'appréhender la valeur esthétique d'un paysage pour en évaluer sa qualité exceptionnelle ; d'autres critères doivent également être pris en compte, qu'il s'agisse de la valeur culturelle, écologique ou même économique (Panizza et Piacente, 1993, 2003 ; Reynard, 2005b). Par exemple, l'aspect grandiose de certaines formes comme les cheminées de fée et le «mystère » apparent qui tourne autour de leur formation constituent un attrait touristique notable. Lorsque la présence d'une espèce ou d'un habitat est dépendante du maintien des conditions géomorphologiques du site, l'attrait écologique fait aussi partie prenante de la valorisation d'un géomorphosite. Ce dernier est également un lieu pédagogique où la formation de la Terre, l'évolution du climat et de la vie peuvent être mieux comprises (Grandgirard, 1997 ; Panizza, 2001 ; Reynard, 2004). Les réseaux hydrographiques abritent de tels lieux, certains étant parfois spectaculaires : gorges, cascades, secteurs en tresses, marais, tourbières, bras morts. Ainsi, pour le scientifique, un tronçon dont le lit est latéralement mobile ou en tresses est synonyme de fortes potentialités écologiques (Ward et al., 2001 ; Florsheim et al., 2008) alors que l'amateur de sensation forte préférera les secteurs en gorges, où il pourra s'adonner aux sports aquatiques dans un cadre naturel remarquable.

\section{Un inventaire exhaustif des géomorphosites}

Aussi exceptionnels qu'ils soient, ce type de sites n'est pas à l'abri de dégradations. Leur préservation tout autant que leur valorisation constituent de facto un enjeu en termes de gestion patrimoniale. Néanmoins, les pratiques de gestion 
ne peuvent être planifiées ni priorisées de manière cohérente que si ces sites ont été préalablement recensés. Pour y parvenir, il est possible de recourir aux données préexistantes. Par exemple, les sites inventoriés dans un guide touristique comme « valant le voyage » (définition donnée par le Guide vert Michelin ; Michelin, 2004) peuvent être considérés comme des géomorphosites ayant une valeur touristique. On peut également recourir aux documents cartographiques, comme les cartes topographiques, ou procéder à des prospections et une analyse géomorphologique in situ afin de repérer les sites potentiels. Cependant, la réalisation d'un inventaire exhaustif sur une aire géographique étendue peut se révéler longue et coûteuse. A l'échelle d'un grand bassin hydrographique comme celui du Rhône, il faudrait parcourir des milliers de kilomètres, ce qui prendrait alors des années, ou sélectionner seulement quelques secteurs, ce qui limiterait alors la portée de la démarche. Il faut donc des outils capables de renseigner de façon automatique ou semi-automatique la structure morphologique des tronçons fluviaux à petite échelle. De plus, pour des raisons opérationnelles, ces outils doivent être transposables d'une région à l'autre. En effet, il est intéressant que l'inventaire des géomorphosites potentiels, bien qu'étant réalisé sur de vastes territoires, repose sur les mêmes critères. Or, depuis une quinzaine d'années, des avancées importantes ont été réalisées en télédétection et en géomatique. Cela concerne non seulement les résolutions spectrales ou spatiales des images mais aussi leurs modes de traitement qui sont de plus en plus diversifiés. Ces améliorations permettent de renouveler la réflexion méthodologique concernant l'automatisation des techniques, la définition des métriques, les requêtes d'analyse spatiale via la géomatique à l'échelle d'un bassin hydrographique de plusieurs milliers de kilomètres. Ces progrès laissent entrevoir des pistes prometteuses en vue de la caractérisation physique d'un réseau hydrographique et ce, à trois échelles distinctes : 1) à l'échelle locale, les améliorations en termes de résolution spectrale et spatiale permettent d'extraire une information de haute qualité en limitant les collectes de données in situ (Lejot et al., 2007) ; 2) à l'échelle d'un bassin hydrographique de quelques milliers de kilomètres carrés, l'automatisation des méthodes d'extraction des informations permet d'obtenir rapidement des résultats homogènes sur l'ensemble du bassin (Reinfelds et al., 2004); 3) à l'échelle du territoire métropolitain, les sources de données disponibles sont nombreuses et variées et couvrent, pour certaines d'entre elles, ce territoire avec une résolution acceptable (http://www.ign.fr/).

\section{Les objectifs}

L'objectif principal de cet article est de présenter une méthode semi-automatique d'identification des géomorphosites potentiels le long du réseau hydrographique. Il s'agit de mettre en place un procédé permettant d'effectuer un inventaire de sites qui, selon des critères morphologiques, pourront être assimilés à des géomorphosites. Par définition, les géomorphosites doivent leur caractère spectaculaire à l'appréciation de l'Homme (raisons culturelles, sociétales ou économiques). Notre objectif ici n'est pas de dresser la liste ni de définir la nature des géomorphosites fluviaux mais de présenter une méthodologie permettant de les détecter. Il ne s'agit pas d'une démarche complète, allant de la caractérisation des paramètres morphologiques à la qualification finale de géomorphosites, mais d'un outil d'aide à la localisation de sites potentiellement exceptionnels à l'échelle du bassin hydrographique. C'est la première étape vers l'identification des géomorphosites, permettant d'envisager par la suite des politiques de planification des actions de préservation et de valorisation des paysages fluviaux de qualité. Pour y parvenir, nous proposons deux exemples basées sur l'utilisation de données numériques rastérisées et vectorisées (BD Ortho ${ }^{\circledR}$, BD Topo ${ }^{\circledR}$ ) et l'extraction de métriques permettant la création d'indicateurs de sélection des géomorphosites fluviaux. Deux types de géomorphosites fluviaux potentiels ont été sélectionnés afin d'asseoir la démarche : 1) les secteur de gorges, qui correspondent à une morphologie de vallée souvent spectaculaire et touristiquement attractive, à l'image de certains tronçons du Verdon et de l'Ardèche. Pour des raisons esthétiques, scientifiques, socio-économiques voire culturelles, les gorges peuvent être assimilées à des géomorphosites ; 2) les tronçons fluviaux présentant un potentiel écologique, qui s'exprime par des propriétés paysagères particulières partiellement intégrées par le style fluvial (présence d'un corridor naturel large bien préservé ; contact complexe entre les milieux terrestres et les milieux aquatiques ou forte mobilité en plan du lit). Au sein de la plaine alluviale, la présence d'écotones sur une longueur significative est un indicateur de la qualité écologique des corridors fluviaux (Naiman et Décamps, 1997 ; Ward et al., 1999 ; Paetzold et al., 2005). De même, les secteurs en tresses, qui doivent leur originalité physique et biologique aux conditions d'apports et de transfert hydrosédimentaires, sont des milieux fluviaux très dynamiques encore peu étudiés, et pourtant caractérisés par une forte diversité biologique, comme cela a été souligné par les travaux effectués sur le Rhône (Bravard et al., 1986), le Tagliamento (Arscott et al., 2000, 2002), le Val Roseg (Ward et Uehlinger, 2003) et la Drôme (Dufour et al., 2007).

\section{Le bassin de la Drôme}

La mise en place de cette méthode nécessite une série de tests sur un linéaire fluvial. Le tronçon principal de la Drôme, long de $106 \mathrm{~km}$, draine un bassin-versant de $1640 \mathrm{~km}^{2}$ (fig. 1). Il est caractérisé par un régime hydrologique préalpin et un climat méditerranéen. Ce bassin a été choisi comme secteur d'études car il se caractérise par des milieux fluviaux très contrastés, avec notamment des secteurs de tressage, endigués ou pas, des gorges et des tronçons rectilignes (Piégay et Landon, 1997). De plus, les liens fonctionnels existant entre la rivière et sa plaine alluviale sont à l'origine d'écosystèmes de qualité. Aussi bien d'un point de vue physique que biologique, le système drômois offre ainsi une diversité paysagère qui en fait un objet d'étude intéressant. 


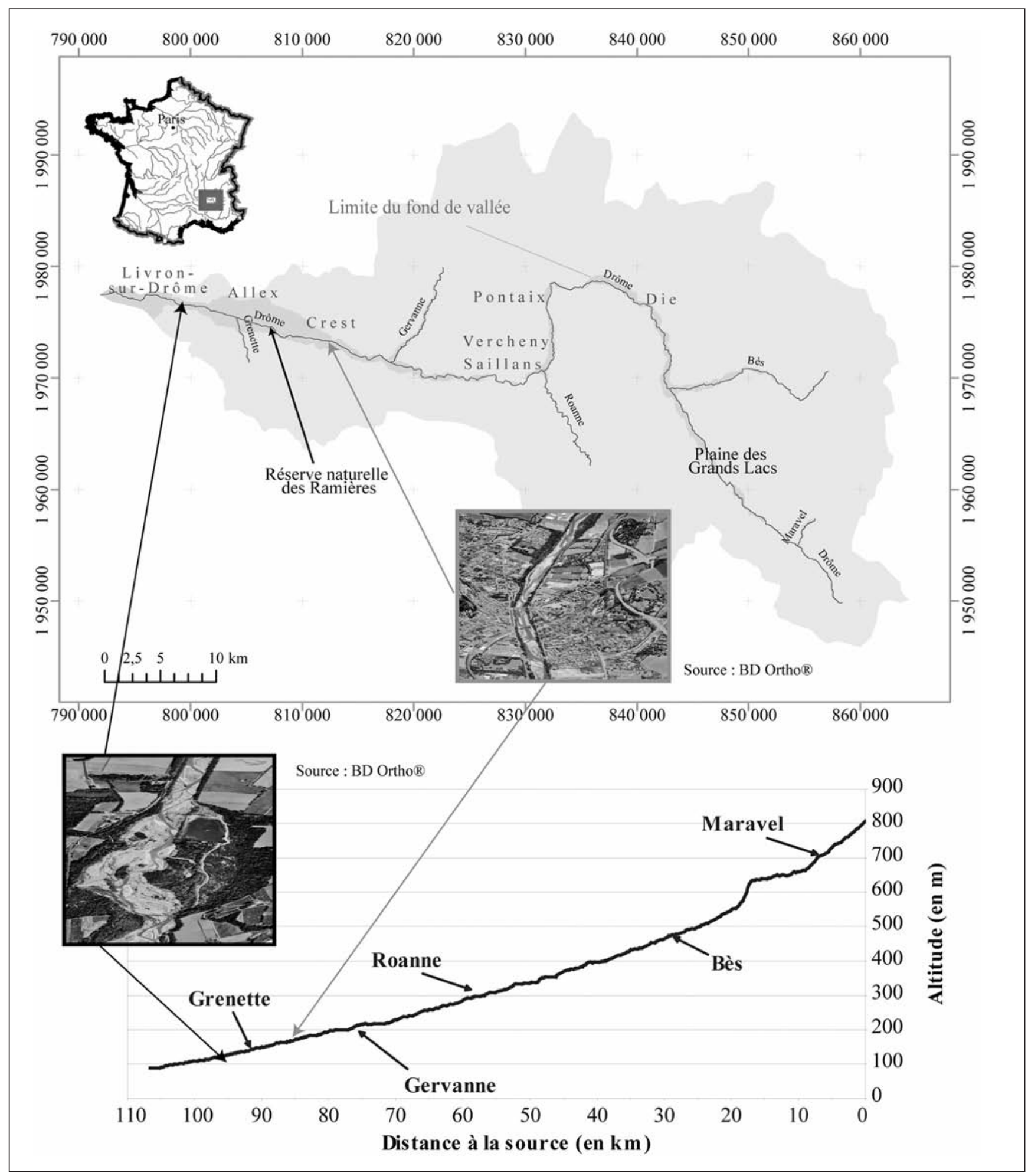

Fig. 1 - Présentation du linéaire principal de la Drôme (variation longitudinale de la largeur du fond de vallée et de la pente).

Fig. 1 - General characteristics of the Drôme main stem (longitudinal variations of valley-bottom width and energy slope).

\section{Données et méthodes}

\section{Données brutes}

La détection des secteurs en gorges ainsi que des tronçons à haut potentiel écologique s'est faite à partir de données rastérisées et vectorisées (tab. 1). Nous avons extrait de la BD Topo ${ }^{\circledR}$ de l'IGN (Institut Géographique National) un MNT (Modèle Numérique de Terrain) au pas de $25 \mathrm{~m}$, permettant notamment l'identification du fond de vallée. La BD Carthage $^{\circledR}$ de l'IGN représentant le linéaire fluvial a également été utilisée sous la forme d'une couche vectorielle. 


\begin{tabular}{|ll|}
\hline BD Carthage & $\begin{array}{l}\text { Référentiel hydrographique } \\
\text { Données vectorielles } \\
\text { Couche hydrographique }\end{array}$ \\
\hline BD Ortho & $\begin{array}{l}\text { Orthophotographie } \\
\text { Résolution spatiale }: 50 \mathrm{~cm} \\
\text { Résolution spectrale : rouge, vert, bleu }\end{array}$ \\
\hline Modèle Numérique de Terrain & Données rastérisées \\
& Résolution spatiale : $25 \mathrm{~cm}$ \\
& Vecteur de précision métrique
\end{tabular}

Tab. 1 - Données brutes issues des bases de données géographiques de l'Institut Géographique National (IGN).

Tab. 1 - Raw data extracted from the geographic databases of the National Geographical Institute (IGN).

Quant à la BD Ortho ${ }^{\circledR}$, elle a servi à extraire sous forme vectorielle les objets composant le corridor alluvial (eau, bancs de galets et végétation riveraine). Cette base de données correspond à une série de photographies aériennes à très haute résolution spatiale $(50 \mathrm{~cm}$ ) rectifiées géométriquement (Wiederkehr et al., 2009), répondant aux différentes exigences requises pour une étude hydromorphologique à petite échelle (Wiederkehr et al., 2008) : une couverture homogène couvrant tout le territoire français avec une résolution spatiale permettant de caractériser un réseau hydrographique déjà conséquent. Malgré cela, la BD Ortho ${ }^{\circledR}$ présente quelques inconvénients, comme la variation de luminosité entre les dalles, ces dernières correspondant aux plaques photographiques $(1 \mathrm{x}$ $1 \mathrm{~km}$ ou $5 \times 5 \mathrm{~km}$ ) vendues par l'IGN. Pour pallier le problème des écarts de luminosité entre les dalles, nous avons procédé en deux étapes. Dans un premier temps, la distribution radiométrique a été homogénéisée pour chaque dalle par le biais d'un étirement radiométrique (à partir de la fonction « interactive stretching » du logiciel ENVI développé par la société ITT). Dans un second temps, un ajustement dynamique a été effectué entre plusieurs images. La radiométrie de l'image à corriger a été ajustée à l'image de référence (fonction «mosaïquage » du logiciel $E N V I$ ). L'autre inconvénient de la BD Ortho ${ }^{\circledR}$ est sa taille. En effet, la quantité de données nécessaire pour couvrir par exemple le bassin hydrographique du Rhône est de l'ordre de deux téraoctets. Une telle masse de données pose des problèmes de stockage mais également de temps de traitement.

\section{Mise en forme des données}

Construction d'un fond de vallée vectoriel. A l'instar d'A. Alber et H. Piégay (sous presse), nous avons considéré le fond de vallée comme l'objet géographique correspondant au sommet du remblaiement alluvial holocène, également nommé la plaine alluviale. Assimilée à l'espace maxi- mal au sein duquel le cours d'eau peut potentiellement se déplacer, inonder et/ou alluvionner (Piégay et al., 2005a), la plaine alluviale est une entité clé pour la caractérisation de nombreux géomorphosites. L'objet vectoriel représentant le fond de vallée a été construit à partir du modèle numérique de terrain issu de la $\mathrm{BD}$ Topo $^{\circledR}$ de l'IGN et de la couche vectorielle extraite de la BD Carthage ${ }^{\circledR}$. Il s'agit d'assimiler le fond de vallée à l'espace inondable pour une hauteur d'eau en lit majeur dont le seuil est une valeur constante (Williams et al., 2000) ou une fonction de la taille du cours d'eau et d'un débit de référence (Dodov et Foufoula-Georgiou, 2006 ; Hall et al., 2007). Nous avons extrait le réseau hydrographique de la BD Carthage ${ }^{\circledR}$ et appliqué une statistique zonale sur ce réseau pour créer sous forme rastérisée un plan de référence altimétrique. Le MNT de la BD Topo ${ }^{\circledR}$ a été soustrait à ce plan de référence afin de construire un MNT relatif. Ont alors été extraites les cellules dont la valeur était inférieure au seuil fixé de manière empirique à $10 \mathrm{~m}$ dans les zones montagneuses, et à $5 \mathrm{~m}$ dans les zones à faible relief. Finalement, cette couche raster a été convertie en entité vectorielle. Nous avons utilisé le fond de vallée comme masque afin d'extraire une couverture rastérisée issue de la BD Ortho ${ }^{\circledR}$ de l'IGN couvrant uniquement ces espaces. A partir de la nouvelle couche vectorielle, les « grands » types de plaine alluviale, comme le type « confiné », ont pu être identifiés (Alber et Piégay, sous presse).

Identification des objets géographiques unitaires par classification orientée-objet. Trois classes d'objets composant le corridor alluvial (eau, bancs de galets et végétation riveraine) ont été extraites sous forme vectorielle et rastérisée à partir des images représentant les fonds de vallée. L'extraction a été réalisée à partir d'une classification orientée-objet (module « Feature Extraction », logiciel ENVI) ; l'objet a été décrit non seulement par sa valeur radiométrique mais également par sa forme, sa texture et son contexte, ce qui est un complément important dans le cas d'image à faible résolution spectrale. La classification orientée-objet a été décomposée en deux étapes (Wong et al., 2003) : la segmentation puis la classification stricto sensu des objets. La fonction d'extraction utilisée a appliqué une logique floue, c'està-dire que le classificateur a attribué à chaque objet une classe en fonction du degré d'appartenance (ou de probabilité) à chaque classe (Thomas, 2005). Les règles de classification ont été obtenues et appliquées en tant que classification experte. Réalisée avec le logiciel $E N V I$, l'étape de segmentation a été décomposée en trois sous-étapes. Dans chaque cas, des tests visuels ont été effectués afin d'identifier les seuils de meilleure segmentation. La première sousétape a été l'ajustement du niveau d'échelle pour délimiter les frontières des objets et la taille des zones homogènes. Nous avons opté pour un niveau de 40 sur un total de 100. La seconde sous-étape a été le paramétrage du niveau de fusion des objets. Cette sous-étape a permis de résoudre la question de la sur-segmentation de l'image, en regroupant les objets contigus présentant des valeurs radiométriques quasi-identiques. Nous avons choisi un niveau de 96 sur 
100. La troisième sous-étape a permis d'affiner la segmentation ; il s'est agi d'un seuillage qui a regroupé les segments adjacents ayant une luminosité similaire. Dans notre cas, les tests effectués lors de cette étape n'ont montré aucun gain significatif. La classification des objets de l'image a été faite en trois étapes : 1) création d'un jeu de données de références pouvant être assimilé à un observé de terrain, la nature des polygones étant interprétée. Pour cela, une photo-interprétation a été réalisée sur quatre zones tests composées de 1000 polygones (364 bancs de galets, 268 zones en eau et 368 taches de végétation riveraine). Une fois l'étape de photo-interprétation terminée, une trentaine de métriques fondées sur les caractères radiométriques et géométriques des polygones (métriques de forme, de taille, de texture ou de couleur) a été extraite de l'image afin de décrire chacun de ces polygones de référence ; 2) sélection des métriques (tab. 2) et des seuils qui discriminent le mieux des classes de polygones au sein de la base de données créée précédemment et en s'appuyant sur un arbre de segmentation (logiciel $S P S S^{\circledR}$ ) ; 3) application de la classification, en définissant les nouvelles fonctions d'appartenance, qui respecte les seuils définis précédemment. Afin d'améliorer le résultat, un nettoyage manuel de la zone classifiée a été effectué. Nous avons lancé la classification sur deux sites expérimentaux. Les résultats obtenus ont été comparés aux résultats d'une classification manuelle par le biais d'une matrice de confusion (tab. 3). Les résultats montrent que de manière générale, $80 \%$ de la surface a été correctement classée. Les bancs de galets et les taches de végétation ont enregistré des taux de classement

\begin{tabular}{|c|c|c|c|c|}
\hline \multicolumn{2}{|c|}{ Paramètres } & Bancs de galets & Eau & Végétation \\
\hline Forme & Superficie & $\mathrm{X}$ & $\mathrm{X}$ & $\mathrm{X}$ \\
\hline \multirow{3}{*}{ Spectral } & Moyenne - bande 1 & $\mathrm{X}$ & $\mathrm{X}$ & \\
\cline { 2 - 5 } & Moyenne - bande 3 & $\mathrm{X}$ & $\mathrm{X}$ & $\mathrm{X}$ \\
\cline { 2 - 5 } & Ecart-type - bande 2 & & $\mathrm{X}$ & $\mathrm{X}$ \\
\hline Texture & Taux moyen & $\mathrm{X}$ & & $\mathrm{X}$ \\
\hline
\end{tabular}

Tab. 2 - Présentation des métriques retenues pour effectuer la classification.

Tab. 2 - Presentation of the parameters used for classification.

\begin{tabular}{|l|c|c|c|c|}
\hline & \multicolumn{3}{|c|}{ Observation } & \multirow{2}{*}{$\begin{array}{c}\text { Total } \\
\left(\mathbf{e n ~}^{\mathbf{2}}\right)\end{array}$} \\
\cline { 2 - 4 } & $\begin{array}{c}\text { Bancs } \\
\text { de galets }\end{array}$ & Eau & Végétation & \\
\hline \multicolumn{5}{|c|}{ Prédiction } \\
\hline Bancs de galets & $\mathbf{4 1 5} \mathbf{4 8 5}$ & 6040 & 8925 & 430450 \\
\hline Eau & 55232 & $\mathbf{3 2 2} \mathbf{8 8 0}$ & 66880 & 444992 \\
\hline Végétation & 19560 & 321083 & $\mathbf{1 2 1 1 4 1 4}$ & 1552057 \\
\hline Total (en $\left.\mathrm{m}^{2}\right)$ & 490277 & 650003 & 1287219 & 2427499 \\
\hline Erreur d'omission* & $84,7 \%$ & $49,7 \%$ & $94,1 \%$ & \\
\hline Erreur de comission & $96,5 \%$ & $72,6 \%$ & $78,1 \%$ & \\
\hline Taux de classement général & $80,3 \%$ & & & \\
\hline
\end{tabular}

${ }^{*}$ Erreur d'omission : indicateur de non-classement des objets. Le classificateur exclut incorrectement des objets d'une classe.

** Erreur de comission : indicateur d'une mauvaise affectation de classes par classificateur.

Tab. 3 - Matrice de confusion de la classification.

Tab. 3 - Confusion matrix of classification. relativement élevés alors que pour l'eau, près d'un polygone sur deux n'a pas été correctement identifié, la confusion étant faite avec certaines zones de culture.

\section{De l'objet géographique à l'extraction de métriques}

Des tronçons homogènes d'un point de vue géomorphologique ont été identifiés afin de disposer d'un jeu d'entités géographiques ayant une signification physique et sur lesquels produire des métriques et des statistiques associées (Clément et Piégay, 2003). Pour cela, nous avons utilisé deux couches vectorielles : la bande active et le fond de vallée. La largeur de la bande active a été calculée par unités spatiales de $10 \mathrm{~m}$ de long alors que la largeur du fond de vallée, longitudinalement moins variable, a été extraite par unités de $250 \mathrm{~m}$ (Alber et Piégay, sous presse). La variabilité longitudinale de la largeur du fond de vallée, qui est un indicateur de l'organisation géologique du bassin, a permis notamment de distinguer les tronçons alluviaux des tronçons non alluviaux. La largeur de la bande active a permis quant à elle de différencier les zones à chenal unique des zones plus larges présentant de vastes surfaces en galets, exondées à l'étiage. Une fois ces données acquises sur l'ensemble du linéaire drômois, un test de rupture de Pettitt (Pettitt, 1979) a été appliqué sur chacune des deux séries afin d'identifier des tronçons homogènes en moyenne en termes de largeur de fond de vallée d'une part et de largeur de bande active d'autre part. Il s'agit d'un test non paramétrique qui est une variante du test de Mann-Whitney, conçue à l'origine pour deux échantillons distincts. La statistique $U_{\mathrm{d}, \mathrm{N}}$ suppose que pour chaque distance $d$ avec une valeur entre 1 et $N$, les deux séquences longitudinales $\left(X_{\mathrm{i}}\right.$ et $\left.X_{\mathrm{j}}\right)$ pour $i=1$ à $d$ et pour $j=$ $d+1$ à $N$ appartiennent à la même population. $\mathrm{La}$ variable qui doit être testée est le maximum, en valeur absolue, de la variable $U_{\mathrm{d}, \mathrm{N}}$. L'absence de rupture dans la série $\left(X_{\mathrm{i}}\right)$ de taille $N$ est l'hypothèse nulle. 43 tronçons homogènes en termes de largeur de la bande active et 20 tronçons homogènes en termes de largeur du fond de vallée ont été identifiés. En combinant ces deux séries, 53 tronçons géomorphologiquement homogènes ont été identifiés et utilisés pour la suite de la caractérisation.

Comme dans le cas de la largeur du fond de vallée ou de la bande active, l'extraction des métriques permettant de caractériser les tronçons identifiés précédemment a été réalisée de façon spatialement continue et homogène à partir de segments d'extraction des données, dont la longueur était adaptée à la taille des objets. Les segments utilisés faisaient $10 \mathrm{~m}$ de long pour le chenal en eau et $100 \mathrm{~m}$ de long pour les bancs de galets et la végétation riveraine. En fait, la taille de ces segments devait être plus réduite que la taille des objets initiaux afin d'éviter de prendre en compte plusieurs objets de même nature au sein d'un même segment. Qu'elles soient géométriques 
(surface, largeur ; issues d'une couche vectorisée) ou radiométriques (déterminées à partir des orthophotographies), les métriques ont été extraites à partir de ces segments. Ces métriques ont ensuite été moyennées ou calculées par mètre linéaire afin de décrire chacun des tronçons géomorphologiques. Dans le cas des gorges, le tronçon a été également caractérisé par des buffers (zones tampons), qui sont des segments d'extraction longitudinaux. Ceux retenus avaient une largeur de $10 \mathrm{~m}$ au sein desquels d'autres métriques ont été extraites comme des percentiles d'altitude ou des valeurs de pente. Ainsi, ceci a permis de calculer la hauteur de la gorge, la pente de ses versants, son ouverture sommitale ou encore sa symétrie, autant de métriques permettant de caractériser la valeur du site.

\section{Résultats}

\section{Localisation et caractérisation des secteurs en gorges}

Les secteurs en gorges correspondent à des vallées étroites et profondes aux versants relativement raides (Germaine, 2008). Nous les avons considérés comme des géomorphosites en raison, notamment, de l'aspect spectaculaire de leur forme, expliquant l'attrait qu'ils exercent sur les touristes. Il s'agit dans cette partie de présenter les résultats d'une identification des secteurs en gorges, servant de présélection à des géomorphosites de type gorges. Pour identifier un site en gorges, deux indicateurs ont été retenus : l'étroitesse du fond de vallée et les caractères des versants. Afin de détecter automatiquement les secteurs en gorges, un indice de confinement du fond de vallée a été calculé pour chaque tronçon géomorphologiquement homogène identifié précédemment. Le confinement a été calculé comme le rapport entre la largeur de la bande active et la largeur

Fig. 2 - Localisation des tronçons dont le fond de vallée est significativement étroit et au sein desquels sont identifiés les sites en gorges (IS : indice de sinuosité ; LS : longueur du secteur ; LBA : largeur moyenne de la bande active). 1 : tronçons à largeur de fond de vallée étroite (indice de confinement $>0,3$ ).

Fig. 2 - Localisation of channel reaches characterised by a narrow alluvial valley within which the gorges are identified from a set of metrics describing the valley geometry (IS: sinuosity index; $L S$ : length of sector; $L B A$ : mean width of active band). 1: reaches with valley bottom narrow (confinement index $>0,3$ ).

Secteur 2 du fond de vallée. Plus ce rapport se rapproche de 1, plus la place occupée par le cours d'eau dans le fond de vallée est grande. Cet indice a permis de rendre compte du contrôle exercé par la vallée sur le tracé du cours d'eau. Différentes valeurs de l'indice ont été testées visuellement et la valeur de 0,3 a été retenue (fig. 2). Ainsi, cette étape a permis de localiser les vallées étroites. Une fois cette première sélection faite, la forme des versants et leur hauteur ont été calculées pour chacune des vallées retenues afin de distinguer les gorges proprement dite des vallées en V, largement évasées et parfois faiblement incisées dans les plateaux environnants. Les gorges étant par définition des vallées étroites aux versants escarpés, au sein des secteurs à fond de vallée étroit, la morphologie des versants a été caractérisée à partir des données du MNT synthétisées au sein de buffers dont la largeur est en fonction de la largeur du fond de vallée. Une pente moyenne et une altitude maximale ont été extraites de chacun des buffers (tab. 4). Dans le bassin de la Drôme, un

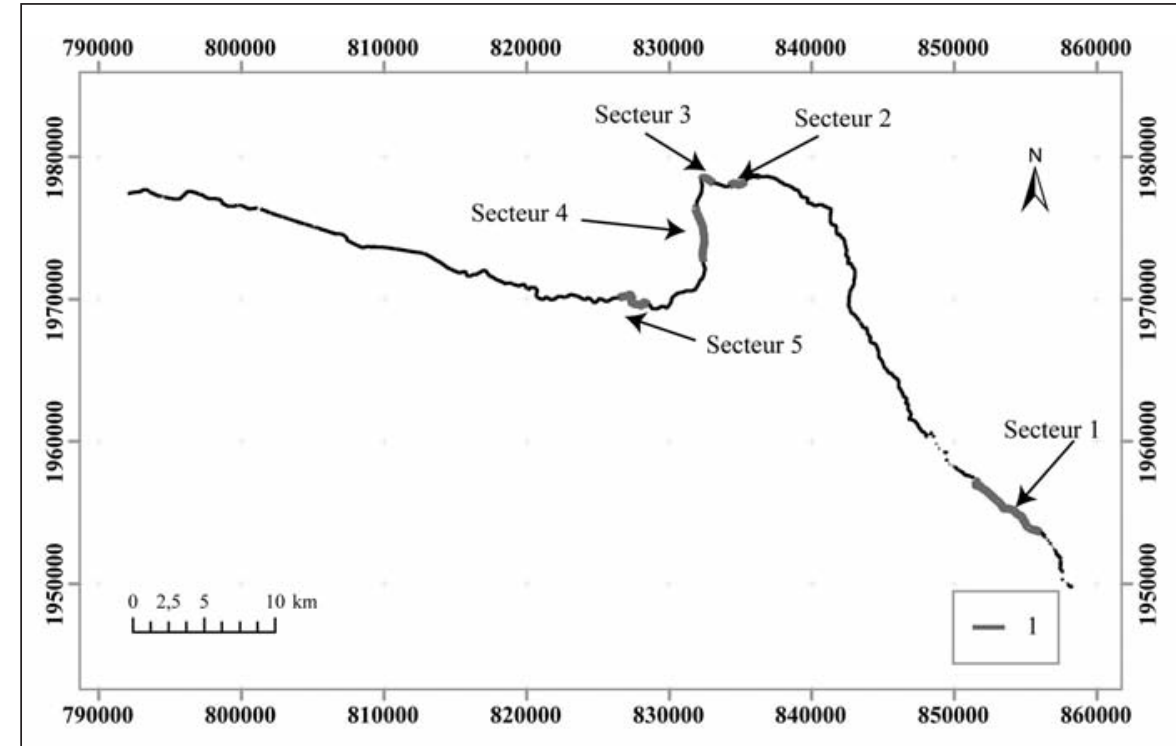

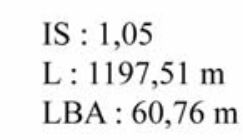

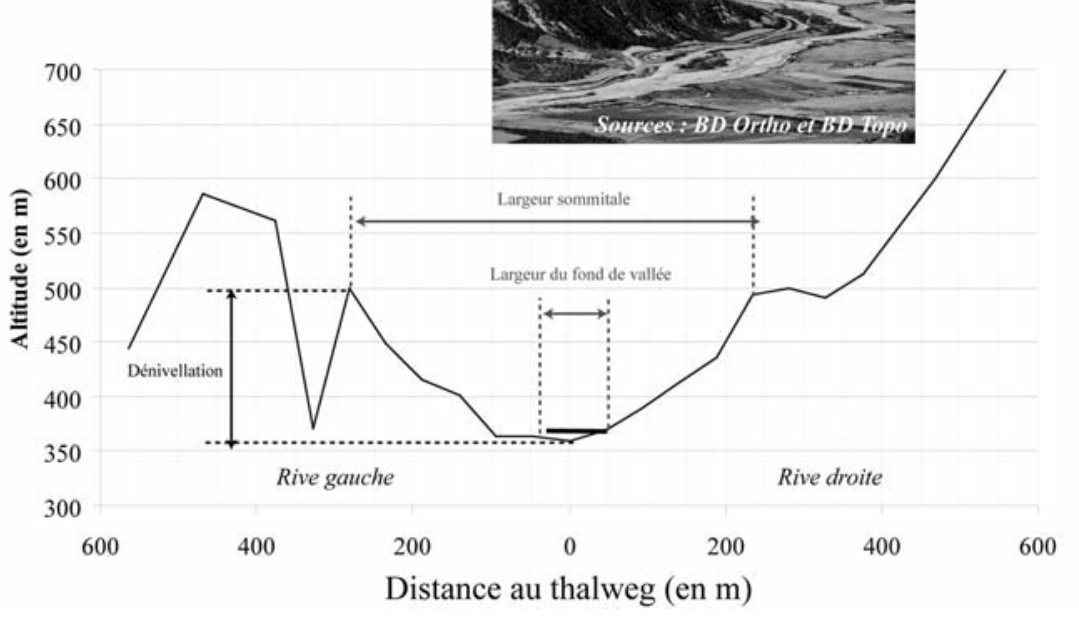




\begin{tabular}{|l|l|c|c|c|c|c|}
\hline & Secteur 1 & Secteur 2 & Secteur 3 & Secteur 4 & Secteur 5 \\
\hline \multicolumn{2}{|l|}{ Largeur moyenne du fond de vallée (en m) } & 125,81 & 187,81 & 277,26 & 367,52 & 178,5 \\
\hline Largeur sommitale (en m) & NR & 704,27 & 554,53 & NR & 401,63 \\
\hline \multicolumn{2}{|l|}{ Indice (fdv/sommet) } & NR & $\mathbf{0 , 2 7}$ & $\mathbf{0 , 5 0}$ & NR & $\mathbf{0 , 4 4}$ \\
\hline \multirow{2}{*}{ Altitude max (en m) } & Rive gauche & NR & 499 & 352 & 399 & 317 \\
\cline { 2 - 7 } & Rive droite & 671 & 499 & 442 & NR & 337 \\
\hline \multicolumn{2}{|l|}{ Altitude moyenne du fond de vallée (en m) } & 670,5 & 361 & 352 & 316,5 & 259,5 \\
\hline \multicolumn{2}{|l|}{ Dénivellation max (en m) } & NR & $\mathbf{1 3 8}$ & $\mathbf{9 0}$ & NR & $\mathbf{7 7 , 5}$ \\
\hline $\begin{array}{l}\text { Pente moyenne } \\
\text { (en \%) }\end{array}$ & Rive gauche & NR & $\mathbf{6 1 , 8 6}$ & $\mathbf{1 6 , 3 5}$ & $\mathbf{3 6 , 8 5}$ & $\mathbf{3 6 , 4 7}$ \\
\cline { 2 - 7 } & Rive droite & $\mathbf{5 2 , 6 5}$ & $\mathbf{5 9 , 8 4}$ & $\mathbf{4 3 , 9 1}$ & $\mathbf{N R}$ & $\mathbf{3 9 , 5 1}$ \\
\hline
\end{tabular}

de secteurs à fort potentiel écologique. Là encore, la démarche de sélection a reposé sur des requêtes établies à partir des caractères planimétriques des taches paysagères. Nous avons retenu deux indicateurs : le style fluvial et la densité d'écotones. Dans le premier cas, parmi tous les styles identi-

Tab. 4 - Présentation des métriques extraites pour caractériser les secteurs à fond de vallée étroit.

Tab. 4 - Presentation of the parameters used to characterise the reaches with narrow valley bottom. fiés, seuls certains comme le tressage ont été considérés comme des indica-

seul tronçon a été qualifié in fine de gorges parmi les cinq secteurs étroits identifiés préalablement. Ce secteur a été décrit à l'aide de plusieurs métriques comme la largeur moyenne de la bande active, la longueur du tronçon, l'indice de sinuosité du fond de vallée et l'évasement de la vallée et sa hauteur (fig. 2).

\section{Localisation et caractérisation des corridors fluviaux à haut potentiel écologique}

Le deuxième exemple de pré-localisation de géomorphosites à partir d'outils géomatiques est centré sur la détection teurs d'un bon potentiel écologique. Dans le second cas, l'indication a été apportée par une forte valeur absolue de la métrique retenue.

Caractérisation par les styles fluviaux. Nous avons mis en place une banque de données rassemblant plusieurs métriques (largeur et longueur de la bande active ou du fond de vallée, surface en eau, etc.) pour chacun des 53 tronçons homogènes déterminés précédemment. La combinaison de certaines de ces métriques a permis de déterminer les styles fluviaux, donc d'identifier les zones à fort potentiel écologique ou, au contraire, celles où l'intervention humaine a

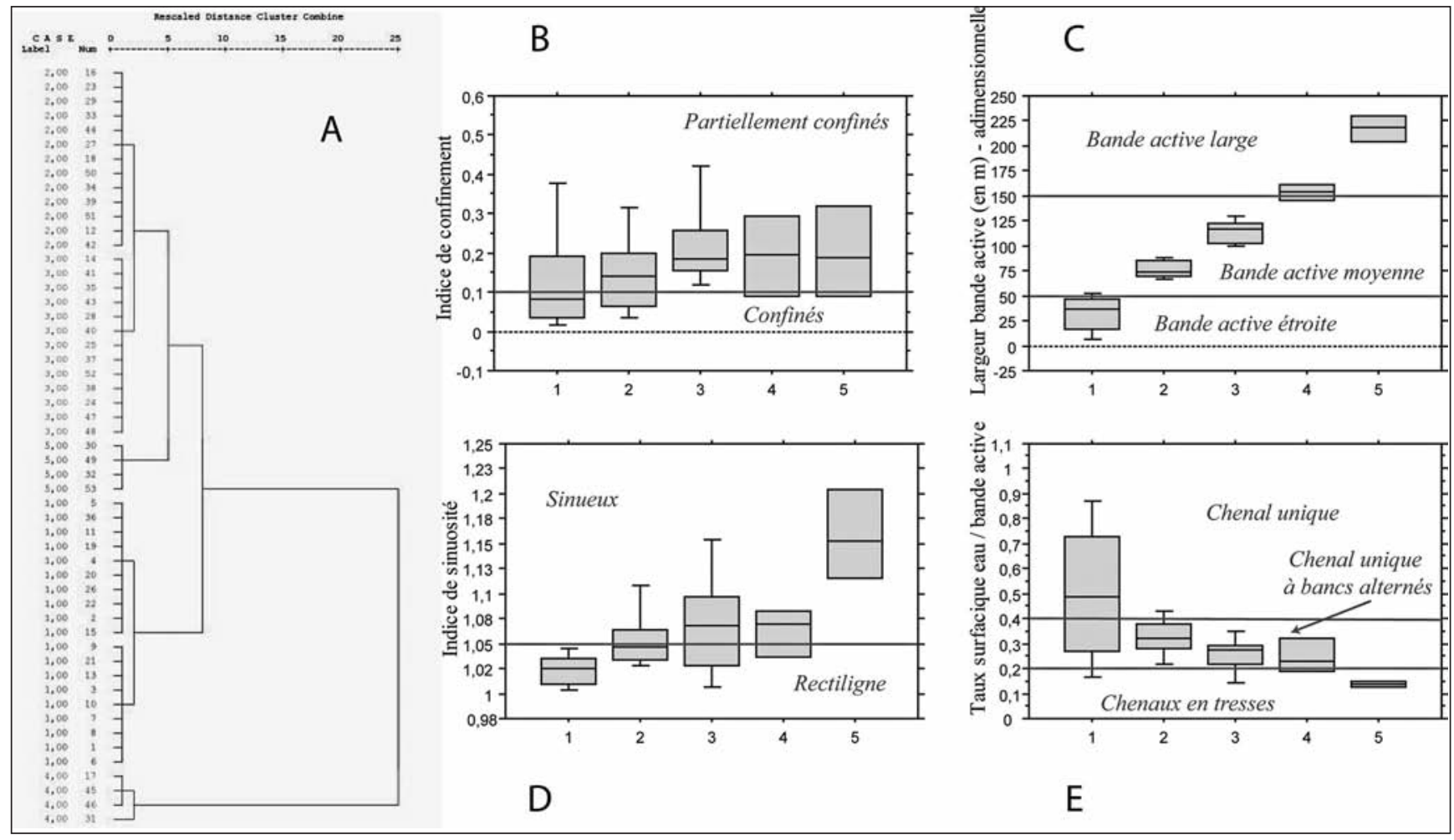

Fig. 3 - Résultat de la classification ascendante hiérarchique réalisée sur 53 tronçons fluviaux caractérisés par quatre métriques géomorphologiques. A : Dendrogramme ordonnant les 53 tronçons et les distributions par classes. B : Indice de confinement. C : Largeur de la bande active. D : Indice de sinuosité. E : Taux surfacique eau/bande active.

Fig. 3 - Result of hierarchical clustering performed on 53 river reaches characterised by four geomorphic metrics. A: Ascending hierarchical classification ordering 53 reaches and distributions per class. B: Box plot, confinement index. C: Box plot, average width of the active channel. D: Box plot, index of sinuosity. E: Box plot, average surface water channel/active channel ratio. 
pu détériorer la qualité du corridor comme, par exemple, les zones endiguées. Les précédents travaux portant sur la détermination des styles fluviaux reposent bien souvent sur le même type de métriques, i.e. planimétriques (Leopold et Wolman, 1957 ; Schumm, 1985 ; Ward et al., 2002 ; Beechie et al., 2006). En s'appuyant sur ces connaissances, nous avons extrait pour chaque tronçon homogène quatre métriques synthétiques résultant pour les trois premières de la moyenne des métriques calculées au niveau des segments d'extraction : 1) la largeur moyenne de la bande active normalisée par la taille du bassin-versant, qui a permis d'appréhender les anomalies locales de la largeur et notamment les secteurs endiguées qui présentaient une largeur très faible pour une taille de bassin donnée ; 2) l'indice moyen de confinement, qui donne une indication sur les possibilités de déplacement latéral du cours d'eau. Cet indice est le rapport de la largeur de la bande active par la largeur du fond de vallée ; 3) la part occupée par le chenal en eau au sein de la bande active, apportant des indications sur l'abondance de la charge solide en transit (Alber et Piégay, sous presse) ; 4) l'indice de sinuosité du cours d'eau, qui est le rapport entre la longueur de l'axe médian de la bande active divisée et la longueur de l'axe médian du fond de vallée. Ce dernier indice ne repose pas sur une moyenne des métriques extraites par segment mais sur deux métriques renseignées à l'échelle du tronçon homogène. Par ailleurs, une classification ascendante hiérarchique (Lebart et al., 1995) a été réalisée sur le jeu de données des 53 tronçons et des quatre variables. La lecture du dendrogramme résultant de cette analyse a permis de distinguer cinq classes, correspondant chacune à un style fluvial particulier allant d'un chenal unique, étroit et sinueux à un lit en tresses. Afin de caractériser chaque style, la distribution statistique (médiane, quartiles) de chacune des métriques a été représentée graphiquement par classe (fig. 3). Une fois cette identification réalisée, les secteurs en tresses considérés comme des lieux à fort potentiel

Fig. 4 - Répartition longitudinale des styles fluviaux le long du cours principal de la Drôme.

Fig. 4 - Longitudinal evolution of geomorphic patterns along the Drôme river main stem. écologique ont pu être localisés, la carte dressée pouvant alors servir d'aide à la reconnaissance de géomorphosites (fig. 4).

Caractérisation par l'approche écotonale. Une autre manière d'identifier les sites à haute valeur écologique a été de quantifier le linéaire de contact entre les taches paysagères, ce que les écologues appellent un écotone. Celui-ci correspond à une zone de transition entre deux écosystèmes. Bien qu'étant parfois une frontière entre deux systèmes pour certaines espèces - par exemple une île qu'un poisson est obligé de contourner pour se rendre d'une rive à l'autre -, l'écotone joue un rôle important dans le maintien de la biodiversité en lien avec une forte spécificité écologique (Naiman et Décamps, 1997). L'intérêt de cette démarche par rapport à la précédente est qu'elle a permis l'identification de sites fondée sur des métriques caractérisant la bande naturelle, sans avoir recours à des données vectorielles caractérisant le fond
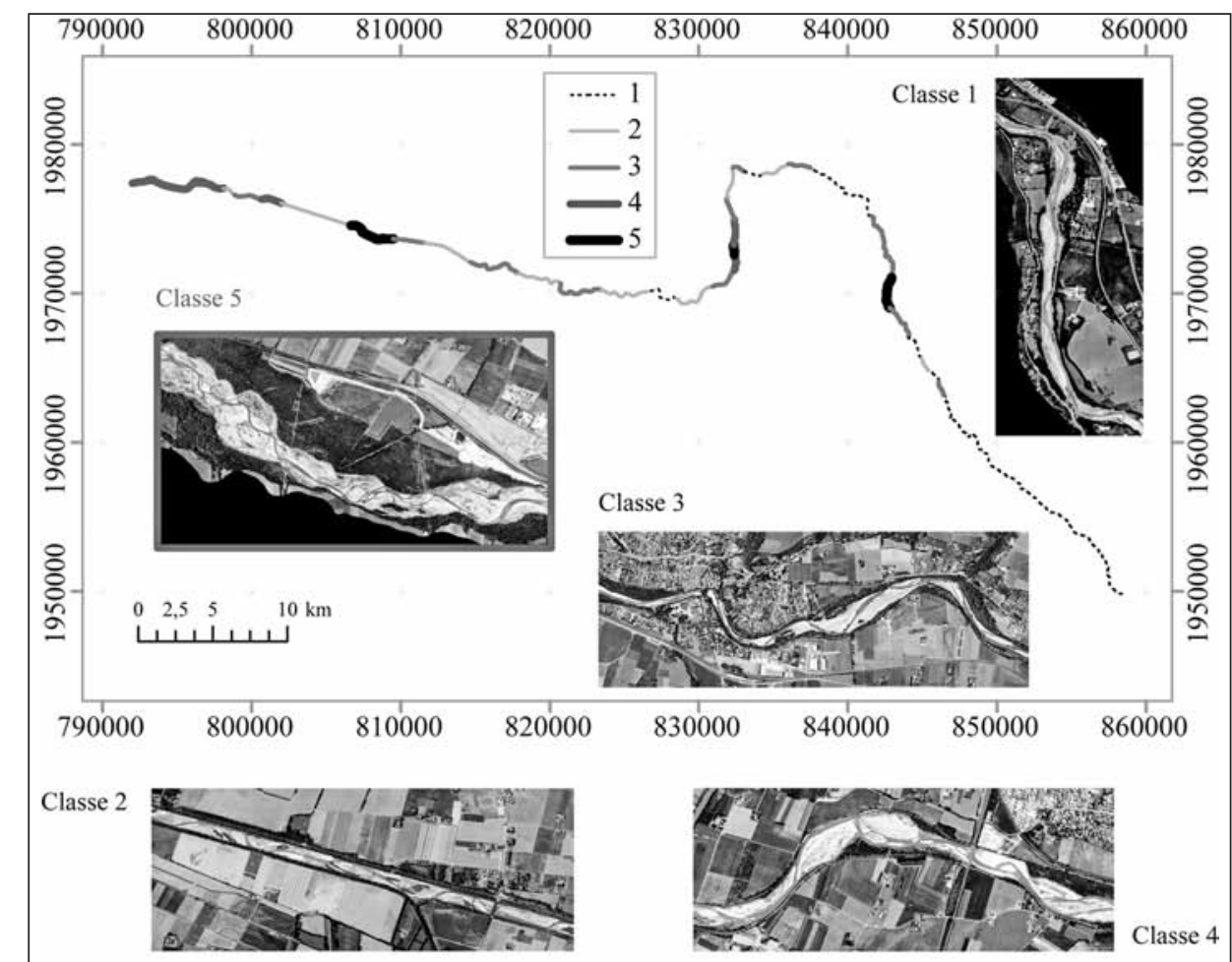

\begin{tabular}{|c|c|c|c|c|c|}
\hline Classes & $\begin{array}{l}\text { Largeur de la } \\
\text { bande active }\end{array}$ & $\begin{array}{l}\text { Indice de } \\
\text { sinuosité }\end{array}$ & $\begin{array}{l}\text { Indice de } \\
\text { confinement }\end{array}$ & $\begin{array}{l}\text { Taux surfacique } \\
\text { eau / bande active }\end{array}$ & Style fluvial \\
\hline $1^{*}$ & Etroite & Rectiligne & Confinés & Unique & Chenal en eau étroit et rectiligne \\
\hline 2 & Moyenne & Rectiligne & $\begin{array}{l}\text { Partiellement } \\
\text { confinés }\end{array}$ & $\begin{array}{l}\text { Unique à bancs } \\
\text { alternés }\end{array}$ & $\begin{array}{l}\text { Chenal rectiligne de largeur } \\
\text { moyenne à bancs alternés }\end{array}$ \\
\hline 3 & Moyenne & Sinueux & $\begin{array}{l}\text { Partiellement } \\
\text { confinés }\end{array}$ & $\begin{array}{l}\text { Unique à bancs } \\
\text { alternés }\end{array}$ & $\begin{array}{l}\text { Chenal divaguant de largeur } \\
\text { moyenne à bancs alternés }\end{array}$ \\
\hline 4 & Large & Sinueux & $\begin{array}{l}\text { Partiellement } \\
\text { confinés }\end{array}$ & $\begin{array}{l}\text { Unique à bancs } \\
\text { alternés }\end{array}$ & $\begin{array}{c}\text { Chenal divaguant à bancs alternés } \\
\text { avec une large bande active } \\
\text { partiellement confinée }\end{array}$ \\
\hline 5 & Large & Sinueux & $\begin{array}{l}\text { Partiellement } \\
\text { confinés }\end{array}$ & Tresses & $\begin{array}{l}\text { Chenaux en tresses avec } \\
\text { une large bande active }\end{array}$ \\
\hline
\end{tabular}


de vallée. Au sein des corridors fluviaux, nous avons identifié trois types d'écotones : contact milieu aquatique/alluvions nues ; contact alluvions nues/milieu arboré riverain ; contact milieu arboré riverain/milieu aquatique. Au sein de chaque tronçon homogène défini précédemment, la longueur cumulée de ces trois types de contact et leur densité (rapport entre la longueur cumulée de contacts et la longueur du tronçon homogène) a été calculée et cartographiée (fig. 5).

\section{Discussion}

\section{Les bases de données info-cartographiques, des sources clés pour une pré-identification des géomorphosites}

Les géomorphosites sont considérés comme des sites exceptionnels, voire grandioses, à plusieurs titres : paysagers, écologiques, économiques, historiques, ou bien touristiques (Panizza et Menella, 2007). Pour valoriser, préserver ou même restaurer de tels sites, les opérateurs publics doivent disposer d'informations cartographiques et de méthodes géomatiques pour les répertorier et les caractériser (Pereira et al., 2007 ; Reynard et al., 2007 ; Zouros, 2007). La démarche d'extraction présentée ici est avant tout méthodologique. Ainsi, nous avons retenu plusieurs sources d'information disponibles qui peuvent se combiner, à savoir un MNT, des couches vectorielles existantes (des polylignes et des polygones) et des orthophotographies. Plusieurs types d'indicateurs ont également été retenus, faisant appel à des procédures de segmentation latérale (buffers) et longitudinale (segments élémentaires) et à des objets linéaires ou surfaciques agrégés, permettant une pré-identification des géomorpho-

Fig. 5 - Cartographie de la densité d'écotones par tronçons homogènes du corridor fluvial de la Drôme. 1 : très faible ; 2 : faible ; 3 : moyenne ; 4 : forte ; 5 : très forte. Méthode : pour chaque tronçon homogène la longueur de contact entre deux écosystèmes est divisée par la longueur du tronçon.

Fig. 5 - Map of the ecotonal density by homogeneous reaches of the river corridor of the Drôme River. 1: very low; 2: low; 3: average; 4: high; 5: very high. Method: for each homogeneous reach the length of contact between two different ecosystems is divided by the length of the reach. sites fluviaux. La méthode peut être facilement généralisée à des linéaires plus étendus et s'appuyer sur d'autres métriques. Pour autant, cette méthode d'identification des sites à fort potentiel patrimonial ne permet pas d'identifier les géomorphosites. En effet, selon M. Panizza et S. Piacente (1993), de tels sites présentent des qualités esthétique, culturelle, écologique ou même économique qui doivent s'apprécier par d'autres voies. Les questions esthétiques pourraient notamment s'intégrer facilement à la présente démarche compte tenu des récents développements proposés par Y.-F. Le Lay et al. (2005) et H. Piégay et al. (2005b). Si à l'heure actuelle, il n'est pas possible de détecter automatiquement les géomorphosites, la détermination de sites potentiels, compte tenu de leurs caractéristiques morphologiques, reste utile car elle offre la possibilité d'une présélection semi-automatique à l'échelle des bassins hydrogra-

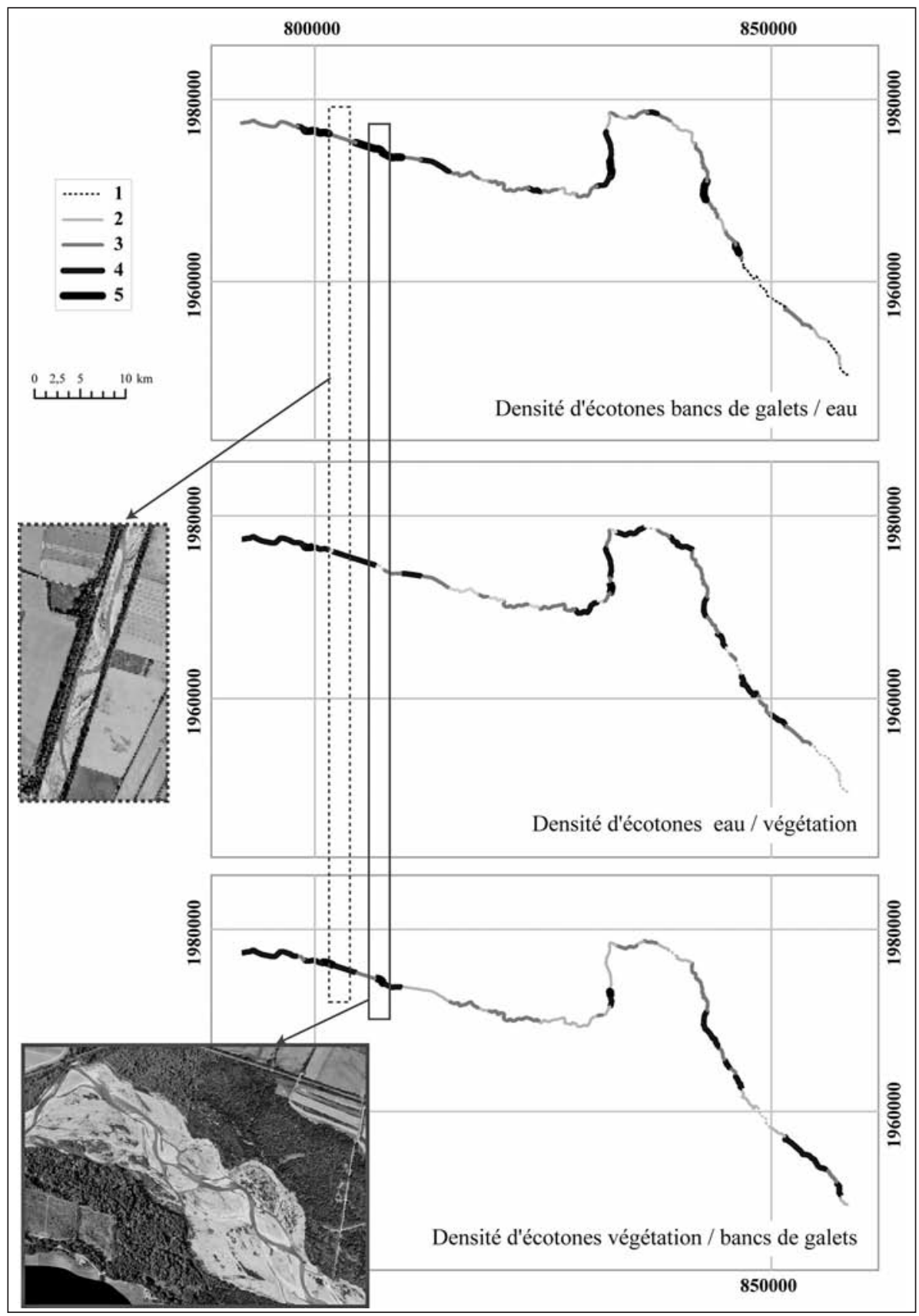


phiques étendus, réduisant d'autant le temps nécessaire ensuite pour les déterminer à partir des critères de M. Panizza et S. Piacente (1993).

\section{Originalité et application de ces outils à d'autres problématiques}

Les enjeux liés à la détermination des géomorphosites sont très proches de ceux qui se posent dans le cadre de la mise en œuvre de la Directive Cadre Européenne sur l'Eau (DCE). En effet, l'application de la DCE implique qu'un état des lieux de toutes les masses d'eau soit réalisé (Wasson, 2001 ; Roche et al., 2004 ; Porcher, 2009), les pays signataires s'engageant à prendre des mesures pour atteindre, d'ici 2015, un bon état écologique des milieux aquatiques. Les mesures pour atteindre ce bon état étant bien souvent hydromorphologiques, il est nécessaire de caractériser l'état physique des cours d'eau. Une fois cet état des lieux effectué, il sera possible de définir un écart à l'état de référence et ainsi privilégier les secteurs à sauvegarder ou à restaurer. C'est dans ce contexte opérationnel que les outils présentés dans cet article ont été développés. Ces outils permettent l'identification automatique (ou semi-automatique) de tronçons à partir de métriques à définir par l'opérateur en fonction de son objectif. La réalisation d'un inventaire qui soit le plus continu possible sur un réseau fluvial étendu semble aujourd'hui possible. Notons enfin que si ces outils peuvent relever d'un usage opérationnel, ils sont également susceptibles d'être utilisés à des fins scientifiques et ouvrent ainsi la voie à l'élaboration de modèles géomorphologiques régionalisés et à l'analyse des liens existant entre une géométrie fluviale donnée et les paramètres de contrôle amont.

\section{L'apport des outils géomatiques}

Constituer une base de données à petite échelle et sans discontinuité longitudinale implique que la procédure soit bien normalisée. Pour cela, les outils géomatiques offrent probablement la solution technique la plus pertinente tant en termes de normalisation que d'efforts financiers et humains pour obtenir cette information. Un des avantages de cette méthode est la possibilité de stocker des données dans un système d'information à référence spatiale et de facilement revenir sur celles-ci. Ainsi, à partir de nouvelles requêtes, il est possible de créer de nouveaux indicateurs construits à partir de la combinaison de différentes métriques de la bibliothèque qui s'enrichit donc de façon cumulative. La mise en place d'une telle démarche nécessite de réaliser initialement une série de tests plus ou moins longs. Si le démarrage est lourd et nécessite une infrastructure informatique importante, la détection des objets géographiques et le calcul de métriques sur un réseau hydrographique de plusieurs milliers de kilomètres offrent ensuite un champ de recherche très large et un outil directement opérationnel. Grâce aux progrès techniques de ces dernières années et la disponibilité d'informations nouvelles, des travaux qui n'étaient pas envisageables il y a encore peu de temps sont maintenant possibles. Les procédures de classifications orientées objets, comme celles proposées par Envi Zoom d'ITT ou eCognition de Definiens, permettent d'atteindre des résultats très satisfaisants en terme de détection des taches paysagères à partir d'images certes relativement pauvres d'un point de vue spectral mais avec une résolution spatiale actuellement inégalée à une telle échelle géographique (Karsenty, 2005 ; Perrez Correa, 2004 ; Geerling et al., 2008). Intégrant la forme de la tache dans la procédure de détection ou en associant cette démarche initiale à des requêtes de sélection intégrant la contigüité des taches - le chenal principal est constitué d'objets « eau » qui se touchent, les bancs de galets jouxtent systématiquement le chenal en eau-, ces procédures automatisées offrent ainsi des résultats proches de ceux résultant d'une photo-interprétation traditionnelle et ce, dans un laps de temps compatible avec une analyse à l'échelle d'un réseau hydrographique de plusieurs milliers de kilomètres.

\section{L'utilisation de l'imagerie en géomorphologie fluviale : un avenir prometteur}

De tels travaux sont relativement nouveaux en géomorphologie. En effet, les travaux géomorphologiques dans le domaine de l'imagerie sont encore à un stade expérimental, cherchant principalement à détecter tel ou tel paramètre bien souvent sur une seule image et en multipliant les techniques d'imagerie (LIDAR, images multispectrales ou hyperspectrales ; Bryant et Gilvear, 1999 ; Puestow et al., 2001 ; Marcus et al., 2003), ou sur un couple d'images d'une scène donnée (photogrammétrie bathymétrique, MNT à haute résolution ; Westaway et al., 2000, 2001 ; Butler et al., 2002 ; Westaway et al., 2003 ; Lejot et al., 2007). Ainsi, la question géographique des structures spatiales à petite échelle n'est pas encore abordée. Quant aux typologies conduites dans le cadre d'études régionalisées (Andriamahefa 1999 ; Reinfelds et al., 2004), rares sont celles qui s'appuient sur des images. En effet, la plupart d'entre elles reposent sur des mesures in situ collectées à partir d'une série de tronçons échantillonnés (Kondolf et al., 2003 ; Schmitt et al., 2007). A. Chandesris et al. (2007) ont récemment mis en place une approche régionalisée dans le cadre du projet SYRAH (Système Relationnel d'Audit de l'Hydromorphologie des cours d'Eau). Mais celle-ci n'utilise pas l'image, qui apporte pourtant des informations intéressantes au niveau de l'identification des habitats piscicoles ou de la végétation.

La présente démarche est encore relativement pauvre en termes de métriques disponibles comparativement aux approches in situ mais elle offre, en revanche, l'avantage de produire une information exhaustive sur tout le continuum fluvial. Cette particularité est notamment essentielle lorsque l'on souhaite faire un travail de recensement ou effectuer des requêtes d'identification de sites spécifiques répartis au sein d'un vaste réseau ou territoire comme c'est le cas pour les géomorphosites. Par ailleurs, d'un point de vue scientifique, cette méthode ouvre un champ de réflexion nouveau dans la mesure où elle permet de détecter les ruptures dans les structures longitudinales, ruptures qui ne sont pas détectables 
lorsque l'information géographique est discrète. Or, ces ruptures sont essentielles dans un cadre typologique dans la mesure où elles correspondent ni plus ni moins aux limites de tronçons géomorphologiquement homogènes. La procédure de désagrégation/agrégation qui a été proposée par A. Alber et H. Piégay (sous presse) permet de faire émerger des structures spatiales qui ont un sens géomorphologique et au sein desquelles on peut alors extraire des métriques utilisables pour les typologies. Ainsi, cette démarche ouvre la voie à des réflexions sur les échelles spatiales pertinentes, leurs interactions et la transférabilité de ces emboitements multi-scalaires d'un ensemble régional à l'autre, l'idée étant qu'une « échelle spatiale pertinente » pour caractériser une forme fluviale dépend non seulement de la taille du système mais également des processus physiques qui le façonnent.

\section{L'utilisation de l'imagerie : des défis encore à relever}

L'extraction d'indicateurs à partir des images présente certaines limites : liées d'une part à la résolution des images et d'autre part aux caractéristiques des systèmes fluviaux. En dessous d'une certaine taille, un objet n'est plus détectable (Bel Hadj, 2001). En effet, selon le théorème de Nyquist-Shannon qui fixe que «le pas minimal utilisable doit être inférieur ou égal à la demi-longueur du plus petit tronçon qui participe à la formation du contour du polygone ", l'objet doit avoir une taille minimale de deux fois celle du pixel. Par conséquent, des problèmes apparaissent en tête de réseau où la détection d'objets longs et étroits (chenal en eau, linéaire ripicole) devient délicate. De fait, afin de renforcer la procédure de classification, celle-ci doit par exemple intégrer la taille des objets à détecter le long du continuum fluvial. Ainsi, des abaques ont été établis liant la largeur du cours d'eau à la taille des objets (eau, bancs de galets et végétation) à identifier (Wiederkehr et al., 2009). Par ailleurs, les orthophotographies sont prises à des heures différentes de la journée, celle-ci étant ensoleillée ou non, et des problèmes d'ombre portée se posent ainsi variablement dans l'espace, d'une dalle à l'autre. Un chenal en eau ombragé présentera une faible radiométrie et se confondra alors bien souvent avec la végétation arborescente riveraine. De fait, la qualité de la classification et de l'information finale sera géographiquement variable. Les conséquences de ce phénomène ne sont pas encore pleinement identifiées. Reste que les premiers tests que nous avons réalisés dans le bassin de la Drôme se révèlent très encourageants. Nous avons pu travailler sur plus de $80 \%$ du linéaire sans discontinuités en remontant $18 \mathrm{~km}$ seulement à l'aval de la source et couvrir ainsi des cours d'eau dont la largeur varie de $3 \mathrm{~m}$ à $150 \mathrm{~m}$. Il est évident que nous ne pouvons disposer de toutes les métriques sur tout le linéaire et les données qui ne peuvent pas être extraites à partir d'images pourront provenir de sources externes. Identifier le linéaire qui ne peut être caractérisé par image constitue en soi une information qu'il serait utile d'avoir. Ce linéaire a d'ores et déjà un sens typologique et fonctionnel, puisqu'il concerne des cours d'eau de petite taille dont le miroir est couvert par la canopée. Le contact entre ce réseau et celui qui est caractérisable est par ailleurs sans doute complexe, discontinu en plan et peut aussi avoir un sens typologique.

\section{Conclusion et perspectives}

De plus en plus, les problèmes liés à la détérioration de l'environnement et à l'épuisement des ressources naturelles préoccupent l'opinion publique, conduisant les instances institutionnelles à multiplier les directives, les lois et les règlements dans le but de préserver ou de restaurer le patrimoine naturel. Cela implique un fort intérêt à pouvoir localiser les sites à caractères exceptionnels comme les géomorphosites. Dans cet article, nous avons mis en avant des développements méthodologiques permettant la localisation semi-automatique de sites pouvant être qualifiés de géomorphosites fluviaux tels que les gorges et les tronçons en tresses ou à fort potentiel écologique. Ces développements méthodologiques permettent d'envisager de nouvelles perspectives de travail non seulement pour le recensement des géomorphosites mais aussi dans le cadre d'autres travaux de caractérisation des milieux fluviaux à grande ou petite échelle. A partir de la méthode présentée, d'autres types de géomorphosites pourraient être abordés, comme les secteurs à forte mobilité latérale, en incluant un paramètre historique. Il s'agirait alors d'étudier le déplacement latéral de la bande active par unités spatiales à des dates différentes. Nous pouvons également envisager de travailler en se focalisant sur l'objet eau, afin de caractériser les habitats piscicoles en vue de l'amélioration des modèles existants (Ginot, 1998 ; Waddle, 2001 ; Lamouroux, 2002), ou sur l'objet ripisylve, afin de développer des modèles régionalisés de répartition des espèces et des habitats.

\section{Remerciements}

Ce travail s'inscrit dans le cadre du programme cadre Agence de l'eau RMC-Zone Atelier Bassin du Rhône (action 10 du programme 2008) et le Projet Européen FORCASTER (IWRnet). L'objectif principal de ces travaux est de produire une caractérisation physique du réseau hydrographique rhodanien pour définir des priorités d'action en matière de restauration des cours d'eau. Nous remercions l'agence de l'eau RMC qui a mis à notre disposition les couches d'information de la BD Ortho ${ }^{\circledR}$ et de la BD Carthage ${ }^{\circledR}$ ainsi que la couche vectorielle du fond de vallée issue de calculs géomatiques réalisés sur la BD Topo ${ }^{\circledR}$. Nous souhaitons remercier également les relecteurs dont les remarques pertinentes ont permis d'améliorer le manuscrit initial. Enfin, nous remercions Mlle Karen Johnstone pour la relecture des textes en anglais.

\section{Références}

Alber A., Piégay H. (sous presse) - Spatial aggregation procedure for characterizing physical structure of fluvial networks: application to the Rhône basin (France). Geomorphology. 
Andriamahefa H. (1999) - Les hydro-écorégions du bassin de la Loire. Morphologie, hydrologie, pressions anthropiques sur les cours d'eau et les bassins versants. Thèse de Doctorat, université Jean-Monnet (Saint-Etienne), Cemagref BEA/LHQ, volume 1,272 p. + annexes.

Arscott D.B., Tockner K., Ward J.V. (2000) - Aquatic habitat diversity along the corridor of an Alpine floodplain river (Fiume Tagliamento, Italy). Archiv für Hydrobiologie 149, 679-704.

Arscott D.B., Tockner K., Van der Nat D., Ward J.V. (2002) Aquatic habitat dynamics along a braided Alpine river ecosystem (Tagliamento River, Northeast Italy). Ecosystems 5, 802-814.

Beechie T.J., Liermann M., Pollock M.M., Baker S., Davies J. (2006) - Channel pattern and river-floodplain dynamics in forested mountain river systems. Geomorphology 78, 124 - 141.

Bel Hadj Ali A. (2001) - Qualité géométrique des entités géographiques surfaciques. Application à l'appariement et définition d'une typologie des écarts géométriques. Thèse de doctorat en sciences de l'information géographique, université de MarneLa-Vallée, 210 p.

Bravard J.-P., Amoros C, Pautou G. (1986) - Impacts of civil engineering works on the successions of communities in a fluvial system. A methodological and predictive approach applied to a section of the Upper Rhône River, France. Oikos 47, 92-111.

Bryant R., Gilvear D.J. (1999) - Quantifying geomorphic and riparian land cover changes either side of a large flood event using airborne remote sensing, river Tay, Scotland. Geomorphology 23, 1-15.

Butler J.B., Lane S.N., Chandler J.H., Porfiri K. (2002) Through-water close range digital photogrammetry in flume and field environments. Photogrammetric Record 17-99, 419-439.

Chandesris A., Malavoi J.-R., Souchon Y., Wasson J.G., Mengin N. (2007) - Le système relationnel d'audit de l'hydromorphologie des cours d'eau (SYRAH CE) : un outil multi-échelles d'aide à la décision pour la gestion des cours d'eau. IngénieriesEau Agriculture et Territoires, 50, 77-80

Clément P., Piégay H. (2003) - Statistics and fluvial geomorphology. In Kondolf M.G., Piégay H. (Eds): Tools in Fluvial Geomorphology. J. Wiley and Sons, Chichester, 597-630.

Dodov B., Foufoula-Georgiou G. (2006) - Floodplain Morphometry Extraction From a High-Resolution Digital Elevation Model: A Simple Algorithm for Regional Analysis Studies. Ieee Geoscience And Remote Sensing Letters 3-3, 410-413.

Dufour S., Barsoum N., Muller E., Piégay H. (2007) - Effects of channel confinement on pioneer woody plant community structure, composition and diversity along the River Drôme (SE, France). Earth Surfaces Processes and Landforms 32, 1244-1256.

Florsheim J. L., Mount J. F., Chin A. (2008) - Bank erosion as a desirable attribute of Rivers. Bisocience 58, 6.

Geerling W., Kater E., Van den Brink C., Baptist M.J., Ragas A. M.J., Smits A.M.J. (2008) - Nature rehabilitation by floodplain excavation: The hydraulic effect of 16 years of sedimentation and vegetation succession along the Waal River, NL. Geomorphology 99, 1-4, 317-328.

Germaine M-A. (2008) - Évaluer la diversité des paysages de vallées. Réalisation d'une typologie en Basse-Normandie. Norois, 7-19.
Ginot V. (1998) - Logiciel EVHA. Evaluation de l'habitat physique des poissons en rivière (version 2.0.). Guide de l'utilisateur. Cemagref Lyon BEA/LHQ et Ministère de l'Environnement, Direction de l'Eau, Paris, France, 176 p.

Grandgirard V. (1997) - Géomorphologie, protection de la nature et gestion du paysage. Thèse de doctorat es sciences, université de Fribourg, 210 p.

Hall J.E., Holzer D. M., Beechie T.J. (2007) - Predicting river floodplain and lateral channel migration for salmon habitat conservation. Journal of the American Water Resources Association 43-3, 786-797.

Karsenty A. (2005) - Classification orientée objet de la perméabilité des sols en zone urbaine à l'aide d'imagerie très haute résolution et de données laser scanner à Curitiba (Brésil). XYZ, 102, 44-48.

Kondolf G.M., Montgomery D.R., Piégay H., Schmitt L. (2003) - Geomorphic classification of rivers and streams. In Kondolf M.G., Piégay H. (Eds): Tools in Fluvial Geomorphology. J. Wiley and Sons, Chichester, 169-202.

Lamouroux N. (2002) - Estimhab: estimating instream habitat quality changes associated with hydraulic river management. Shareware \& User's guide. Cemagref Lyon-Onema, rapport non publié , $21 \mathrm{p}$.

Lebart L., Morineau A., Piron M. (1995) - Statistique exploratoire multi-dimensionnelle. Paris, Dunod, 439 p.

Lejot J., Delacourt C., Piégay H., Trémélo M.L., Fournier T. (2007) - Very high spatial resolution imagery for reconstructing channel bathymetry and topography from an unmanned controlled platform. Earth Surface Processes and Landforms 32, 1705-1725.

Le Lay Y.-F., Piégay H., Cossin M. (2005) - Les enquêtes de perception paysagère à l'aide de photographies : choix méthodologiques et exemples en milieu fluvial. Septièmes Rencontres de Théo Quant à Besançon du 26 au 28 janvier (http://thema.univfcomte.fr/theoq/pdf/2005/LeLay-theoquant05.pdf).

Leopold L.B., Wolman M.G. (1957) - River channel patterns: braided, meandering and straight. Physiographic and hydraulic studies of rivers. USGS Professional Paper, 282-B., US Government Printing Office, Washington DC, 39-85.

Marcus A.W., Legleither C.J., Aspinall R.J., Boardman J.W., Crabtree R. (2003) - High spatial resolution hyperspectral mapping of in-stream habitats, depths, and woody debris in moutain streams. Geomorphology 55, 363-380.

Michelin (2004) - Guide vert Michelin : Provence. Michelin, 408 p.

Naiman R.J., Décamps H. (1997) - The ecology of interfaces: Riparian zones. Annual Review of Ecology and Systematics 28, 621-658.

Paetzold A., Schubert C., Tockner K. (2005) - Aquatic-terrestrial linkages along a braided river: Riparian arthropods feeding on aquatic insects. Ecosystems 8, 748-759.

Panizza M. (2001) - Géomorphosites: Concepts, methods and examples of geomorphological survey. Chinese Science Bulletin 46, 4-6.

Panizza M., Piacente S. (1993) - Geomorphological assets evaluation. Zeitschrift für Geomorphologie N.F., Suppl. Bd. 87, 13-18. 
Panizza M., Piacente S. (2003) - Geomorfologia culturale. Pitagora Editrice, Bologna, $350 \mathrm{p}$.

Panizza V., Mennella M. (2007) - Assessing geomorphosites used for rock climbing. The example of Monteleone Rocca Doria (Sardinia, Italy). Geographica Helvetica 62, 181-191.

Pereira P., Pereira D., Caetano Alver M.I. (2007) - Geomorphosite assessment in Montesinho Natural Park (Portugal). Geographica Helvetica 62, 159-168.

Perrez Correa M. (2004) - Classification orientée objet d'images à très haute résolution spatiale : application à la cartographie de l'occupation des sols le long des cours d'eau. CEMAGREF, mémoire du Mastère SILAT, $43 \mathrm{p}$.

Pettitt A.N. (1979) - A non-parametric approach to the Changepoint Problem. Applied Statistics 28, 126-135.

Piégay H., Landon N. (1997) - Promoting an ecological management of riparian forests on the Drôme River, France. Aquatic conservation: Marine and Freshwater Ecosystems 7, 287-304.

Piégay H., Darby S.A., Mosselmann E., Surian N. (2005a) - The erodible corridor concept: applicability and limitations for river management. River Research and Applications 21, 773-789

Piégay H., Gregory K.J., Bondarev V., Chin A., Dalhstrom N., Elosegi A., Gregory S.V., Joshi V., Mutz M., Rinaldi M., Wyzga B., Zawiejska J. (2005b) - Public perception as a barrier to introducing wood in rivers for restoration purposes. Environmental Management 36-5, 665-674.

Porcher J.-P. (2009) - Le cadre proposé par la Directive Cadre Européenne sur l'eau : outils de mesure et évaluation de l'état des eaux. La Houille Blanche, 3, 41-45.

Puestow T.M., Simms E.L., Simms A., Butler K. (2001) - Mapping of salmon habitat parameters using airborne imagery and digital ancillary data. Photogrammetric Engineering and Remote Sensing 67-3, 309-317.

Reinfelds I., Cohen T.J., Batten P., Brierley G. (2004) - Assessment of downstream trends in channel gradient, total and specific stream power: a GIS approach. Geomorphology 60, 403-416.

Reynard E. (2004) - Geosites. In Goudie A. (Ed.): Encyclopedia of Geomorphology. Routledge, London, 440.

Reynard E. (2005a) - Paysage et géomorphologie : quelques réflexions sur leurs relations réciproques. In Droz Y., MiévilleOtt V. (Eds.) : La polyphonie du paysage. Presses polytechniques et universitaires romandes, Lausanne, 101-124.

Reynard E. (2005b) - Geomorphological sites, public policies and property rights. Conceptualization and examples from Switzerland. Il Quaternario 18/1, 321-330.

Reynard E., Fontana G., Kozlik L., Scapozza C. (2007) - A method for assessing 'scientific' and 'additional values' of géomorphosites. Geographica Helvetica 62, 148-158.

Roche P., Billen G., Bravard J.-P., Decamps H., Pennequin D., Vindimian E., Wasson J.G. (2004) - Les enjeux de recherche liés à la directive-cadre européenne sur l'eau. Comptes Rendus Geosciences, 337, 1-2, 243-267.

Schmitt L., Maire G., Nobelis P., Humbert J. (2007) - Quantitative morphodynamic typology of rivers. A methodological study based on the French Upper Rhine basin. Earth Surface Processes and Landforms 32-11, 1726-1746.

Schumm S.A. (1985) - Patterns of alluvial rivers. Annual Review of Earth and Planetary Sciences 13, 5-27.
Thomas A. (2005) - Application de l'approche orientée-objet à l'extraction de fragments forestiers à partir de scènes Spot. Mémoire du DESS SIGMA, ENSAT, Toulouse, $42 \mathrm{p}$.

Waddle T.J. (2001) - PHABSIM for Windows: User's manual and exercises. US Geological Survey, Fort Collins, CO, 288 p.

Ward J.V., Tockner K., Schiemer F. (1999) - Biodiversity of floodplain river ecosystems : ecotones and connectivity. Regulated Rivers : Research and Management 15, 125-139.

Ward J.V, Tockner K., Uehlinger U., Malard F. (2001) - Understanding natural patterns and processes in river corridors as the basis for effective river restoration. Regulated Rivers Research and Management 17, 311-324.

Ward J.V., Tockner K., Arscott D.B., Claret C. (2002) - Riverine landscape diversity. Freshwater Biology 47, 517-539.

Ward J.V., Uehlinger U. (Eds.) (2003) - Ecology of a Glacial Floodplain. Kluwer, Dordrecht, 320 p.

Wasson J.G. (2001) - Les questions de recherche posées par la Directive Cadre Européenne sur l'Eau : problématique pour les eaux de surface continentales. Hydroécologie Appliquée, 13-1, $1-19$.

Westaway R.M, Lane S.N., Hicks D.M. (2000) - Development of an automated correction procedure for digital photogrammetry for the study of wide, shallow gravel-bed rivers. Earth Surface Processes and Landforms 25-2, 200-226.

Westaway R.M, Lane S.N., Hicks D.M. (2001) - Airborne remote sensing of clear water, shallow, gravel-bed rivers using digital photogrammetry and image analysis. Photogrammetric Engineering and Remote Sensing 67-11, 1271-1281.

Westaway R.M, Lane S.N., Hicks D.M. (2003) - Remote survey of large-scale braided, gravel-bed rivers using digital photogrammetry and image analysis. International Journal of Remote-Sensing 24-4, 795-815.

Wiederkehr E., Dufour S., Piégay H. (2008) - Apport des techniques d'imagerie pour l'étude des réseaux hydrographiques. Synthèse des connaissances et évaluation d'indicateurs de caractérisation. Agence de l'eau RMC-ZABR-CEREGE, rapport non publié, $43 \mathrm{p}$.

Wiederkehr E., Dufour S., Piégay H. (2009) - Caractérisation du corridor naturel alluvial du réseau hydrographique du bassin du Rhône à partir des orthophotographies de l'IGN. Premiers retours d'expérience pour l'élaboration de modèles hydrogéomorphologiques prédictifs. Agence de l'eau RMC-ZABR-CEREGE, rapport non publié, $65 \mathrm{p}$.

Williams W.A., Jensen M.E., Winne J., Redmond R.L. (2000) An automated technique for delineating and characterizing valley-bottom settings. Environmental Monitoring and Assessment 64-1, 105-114.

Wong T.H. Mansor S.B., Mispan M.R. Ahmad N., Sulaiman W.N.A. (2003) - Feature extraction based on object oriented analysis. In Proceedings of ATC 2003 Conference, 20-21 May 2003, Malaysia, 10 p.

Zouros N. (2007) - Geomorphosite assessment and management in protected areas of Greece. Case study of the Lesvos island - coastal geomorphosites. Geographica Helvetica 62, 168-180.

Article soumis le 19 octobre 2009, accepté le 30 mars 2010. 